\title{
Transient Ischemic Attack in Cerebrovascular Accidents
}

\author{
Dr. R. Narmadhalakshmi M.D ${ }^{1}$, Dr. B. Prabakar ${ }^{2}$ M.D ${ }^{2}$ \\ ${ }^{1}$ Associate Professor, Department of General Medicine, Government Chengalpet Medical College and Hospital \\ ${ }^{2}$ Assistant Professor, Department of General Medicine, Government Chengalpet Medical College and Hospital
}

Summary: In Seventy Two patients admitted in Chengalpet Medical College \& Hospital in General Medicine ward during the period January 2015 to October 2015 with acute Cerebrovascular accident, history of TIA was present in $27 \%$ of thrombotic stroke and $7 \%$ of cardio embolic strokes but was absent in all patients with ICH. 92\% of the TIAs lasted less than an hour. Most of the patients developed stroke within two months of the first TIA.

Aim of the Study: To correlate Transient Ischemic Attack and Cerebrovascular accidents.

Keywords : Cerebrovascular accident - Transient Ischemic Attack.

\section{Introduction}

TIAs are episodes of temporary and focal cerebral dysfunction of vascular origin, rapid in onset, variable in duration, commonly lasting from 2 to 15 minutes but occasionally lasting as long as 24 hours (Milliken and Mc Dowell ${ }^{1}$ 1978).It has been observed that about $25 \%$ to $40 \%$ of the patients with TIA will eventually develop cerebral infarction in the subsequent five years period (Milliken and Mc Dowell 1978). While TIAs are uncommon in ICH it was reported to be present in about $9 \%$ to $10 \%$ of ischemic infarction (Davies ${ }^{2}$ et al 1987) Recently the arbitrary duration of TIA as 24 hours is questioned as most of the TIAs tend to last less than an hour (Levy ${ }^{3}$ 1988).

\section{Material And Methods}

In Seventy Two acute Cerebrovascular accident patients (stroke of less than one week) who were admitted in the General Medicine ward were studied. A detailed history of TIA including the time of occurrence, duration, clinical manifestation, recurrence and the latent period between the first TIA and a stroke were studied. A few patients who were unable to give history of loss of consciousness were included in the TIA absent group. CT scan of brain was done in all the 72 patients immediately after admission and the type of stroke (ischemic or hemorrhagic) was noted .Echocardiogram was done in all the ischemic infarction patients.

\section{Results}

The Total number of acute stroke patients studied was 72 which included thrombotic infarction (40) cerebral embolism of cardiac origin (14) and intracerebral haemorrahage (18). History of TIA was present in $27 \%$ of thrombotic infarction, $7 \%$ of cerebral embolism of cardiac origin. The number and duration of each TIA in thrombotic infarction is given in tables I and II. Single TIA was present in 4 and recurrent TIAs in 10 of 14 patients. Stereotypic recurrence was present in 5 of the patients with recurrent TIA. The clinical manifestation of each TIA in the thrombotic infarction is given in Table III. The latent period between the first TIA and the stroke is given in table IV. In the embolism group two patients (7.1\%) had TIA. Both of them had single TIA, in the first, transient hemiparesis lasting for one hour occurring one week prior to stroke and in the second, transient unsteadiness and confusion for five minutes occurring four hours prior to the stroke was present.

Table I: Number Of Tias In Thrombotic Infarction Prior To Stroke

\begin{tabular}{|l|l|l|}
\hline No of TIAs & No of Patients & \\
\hline 1 & 4 & $28.5 \%$ \\
\hline 2 & 3 & $21.42 \%$ \\
\hline 3 & 1 & $7.14 \%$ \\
\hline 4 & 2 & $14.28 \%$ \\
\hline 5 & 2 & $14.28 \%$ \\
\hline 6 & 0 & - \\
\hline 10 & 1 & $7.14 \%$ \\
\hline 12 & 1 & $7.14 \%$ \\
\hline & 14 & \\
\hline
\end{tabular}

Table II: Duration Of Tia In Thrombotic Infarction (Total No 53)

\begin{tabular}{|l|l|l|}
\hline No of TIAs & No of TIA & \\
\hline 1-5 Min & 25 & $47.2 \%$ \\
\hline
\end{tabular}




\begin{tabular}{|l|l|l|}
\hline 10 Min & 9 & $17 \%$ \\
\hline 15 Min & 11 & $20.7 \%$ \\
\hline 30 Min & 3 & $5.7 \%$ \\
\hline 1Hours & 2 & $3.8 \%$ \\
\hline 2Hours & 1 & $1.9 \%$ \\
\hline 4Hours & 1 & $1.9 \%$ \\
\hline 4Hours & 1 & $1.9 \%$ \\
\hline
\end{tabular}

\section{Discussion}

In this series of 72 patients with acute Cerebrovascular accident history of TIA was not present in any one of the 18 patients with intracerebral haemorrhage. Hence in a patient with acute Cerebrovascular accident if there is a history of TIA it strongly favors ischaemic infarction and almost rules out intracerebral haemorrhage. However it must be remembered that very rarely transient neurological deficit is known to occur in chronic subdural hygroma (Dhanaraj ${ }^{4}$ et al 1987). The incidence of TIA in all types in all types of stroke is

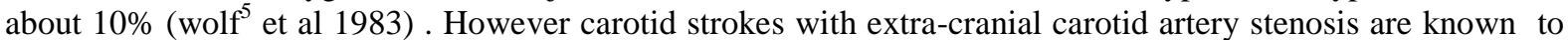
have as high as 50\% to 75\% including of TIA (Mohr ${ }^{6}$ et al 1978). In the present series the incidence of TIA in thrombotic infarction alone was $27.5 \%$. In cerebral embolism of carotid origin it was $7.1 \%$. Both put together it was $24 \%$. Hence even in the Indian population the incidence of TIA is high. Hence it should be possible to prevent stroke at the TIA level itself in a significant percentage of potential thrombotic stroke patients. In this series, when the patients had their first TIA most of them did not consult a physician as they were not aware of the seriousness of the transient symptoms. The public and the family physicians may be educated about the importance of these symptoms since major catastrophes may be avoided by appropriate therapeutic measures. Pr.Dhanraj and Pr. Velmurugendran ${ }^{7}$ et al Studied relation between Transient Ischemic Attack and Stroke.

Table III: Clinical Manifestation Of Tia

\begin{tabular}{|l|l|l|l|}
\hline 1 & Weakness and numbness of one UL & 17 & $32.1 \%$ \\
\hline 2 & Weakness and numbness of one UL and LL & 12 & $22.6 \%$ \\
\hline 3 & Weakness of LL & 7 & $13.2 \%$ \\
\hline 4 & Numbness of one UL and LL & 6 & $11.3 \%$ \\
\hline 5 & Weakness of UL & 5 & $9.4 \%$ \\
\hline 6 & Speech Disturbance & 2 & $3.8 \%$ \\
\hline 7 & Numbness of One LL & 2 & $3.8 \%$ \\
\hline 8 & Weakness of one thumb and index finger & 1 & $1.9 \%$ \\
\hline 9 & $\begin{array}{l}\text { Weakness of and numbness of one UL and LL } \\
\text { UL- Upper Limb } \\
\text { LL- Lower Limb }\end{array}$ & 1 & $1.9 \%$ \\
\hline
\end{tabular}

Table IV: Latency Between The First Tia And Stroke

\begin{tabular}{|l|l|l|}
\hline Latency & Number & \multicolumn{2}{|c|}{ Percentage } \\
\hline 7 days & 5 & $35.7 \%$ \\
\hline $8-14$ days & 2 & $14.3 \%$ \\
\hline $15-30$ days & 2 & $14.3 \%$ \\
\hline $1-2$ months & 3 & $21.4 \%$ \\
\hline 2years & 1 & $7.1 \%$ \\
\hline 5 years & 1 & $7.1 \%$ \\
\hline
\end{tabular}

In general it is said that TIAs are known to last for upto 24 hours. Recently the validity of this duration is questioned by some authors Acheson and Hutchinson ${ }^{8}$ (1964 suggested once hour as the differentiating period between TIAs and stroke. In carotid artery disease. TIAs presenting as transient monocular blindness is known to last less than 15 minutes in most of the patients (Mohr et al 1986). Levy (1988) in his series observed that $60 \%$ of TIAs lasted less than 60 minutes. In the present series the duration of Tia was within 5 minutes in $46.8 \%, 10$ minutes in $65.8 \%, 15$ minutes in $73.4 \%, 30$ minutes in $86 \%$ and one hour in $92.3 \%$. Hence the authors also suggest that while defining the duration of TIA it may be changed to one hour rather than 24 hours.

In the Framingam study, following the first TIA, brain infarction occurred in two thirds of the patients in the first six months and in Rochester, Minnesota, the greatest risk of stroke was within the first month (whisant ${ }^{9}$ et al 1973 wolf et al 1983). In the present study 50\% developed stroke within a week of first TIA (Which includes $27.3 \%$ who developed within 24 hours) $13.6 \%$ developed stroke between 1-2 weeks, $13.6 \%$ between 15-30 days and 13-6\% between 1-2 months. The remaining 9\% developed stroke after two years. This suggest that the potential risk period of developing stroke after the first TIA is two months. The maximal being within one week. Thus every patient who gives history of TIA must be managed carefully for a minimum period of two months as this periods seems to be a highly vulnerable period. 


\section{References}

[1]. Milliken C, McDowell F: Treatment of Trasient ischaemic attacks . Stroke 9, 299-308,1978

[2]. Davies P. Dambrosia J, Schoenberg B et al : Risk factors for ischemic stroke: A prospective study I Rochester, Minnesota.Annuals of Neurology, 22, 319-327,1987

[3]. Levy: How transient are transient ischaemic attacks? Neurology 38, 674-677, 1988

[4]. Dhanaraj, Kumaresan Velmurugendran C U : Chronic subdural Hygroma presenting as transient neurological deficit. Neurology India 35-172, 1987

[5]. Wolf P. Kannel W, Verter J Current status of risk factors of stroke. Neurological clinics of North America . Symposium on cerebrovascular disease. Ed Barnett H J M Saunders Company London 317-343, 1983

[6]. Mohr J Caplan L R Melski J et al : the Harward co-operative stroke registry prospective registry Neurology 28,754-762(1978)

[7]. Pr.Dhanraj and Pr. Velmurugendran Institute of Neurology Government General Hospital Chennai ,Studied related with Transient Ischemic Attack in Stroke

[8]. Acheson J. Hutchinson E.C observation on the natural history of transient cerebral ischaemia. Lancet 2, 871-874,1964

[9]. Whisant J P Matrumoto N Elvebact L R : Transient ischemic attacks in community. Rochesester, Minnesota 1955 through 1969 , Mayo clinic proceedings 48 194-198(1973). 